BNL 50587

(Particle Accelerators and High-Voltage Machines - TID 4500)

\title{
ANOMALOUS LENGTH OF ELECTRON BUNCHES AS AN INSTABIIITY THRESHOLD
}

\author{
E. Messerschmid* and M. Month
}

October 15, 1976

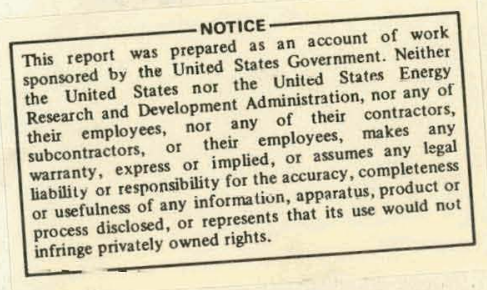

\section{ACCELERATOR DEPARTMENT}

B R O OKHAVEN N A T I ONAL LA B ORA T OR Y A S S OC I A T E D UN IVERS I T I ES, INC.

U P T O N, NEW YOR K 11973

under contract No. EY-76-C-02-0016 with the

UNITED STATES ENERGY RESEARCH AND DEVELOPMENT ADMINISTRATION

* On leave from the University of Freiburg, Freiburg, Germany. 


\section{DISCLAIMER}

This report was prepared as an account of work sponsored by an agency of the United States Government. Neither the United States Government nor any agency Thereof, nor any of their employees, makes any warranty, express or implied, or assumes any legal liability or responsibility for the accuracy, completeness, or usefulness of any information, apparatus, product, or process disclosed, or represents that its use would not infringe privately owned rights. Reference herein to any specific commercial product, process, or service by trade name, trademark, manufacturer, or otherwise does not necessarily constitute or imply its endorsement, recommendation, or favoring by the United States Government or any agency thereof. The views and opinions of authors expressed herein do not necessarily state or reflect those of the United States Government or any agency thereof. 


\section{DISCLAIMER}

Portions of this document may be illegible in electronic image products. Images are produced from the best available original document. 
NOTIC E

This report was prepared as an account of work sponsored by the United States Government. Neither the U.S. nor the U.S.

Energy Researh and Development Administration, nor any of their employees, nor any of their contractors, subcontractors, or their employees, makes any warranty, express or implied, or assumes any legal liability or responsibility for the accuracy, rnmpletencso or usefulness of any information, apparatus, product or process disclosed, or represents that its use would not infringe privately owned Iighls.

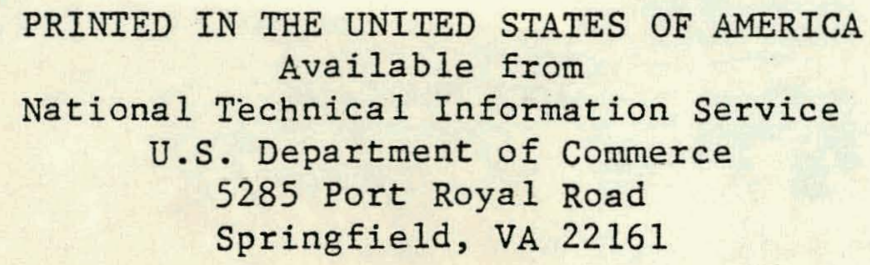

Price: Printed Copy $\$ 3.50$; Microfiche $\$ 3.00$ 


\section{ABSTRACT}

A mechanism. for the anomalous length of electron bunches, based on the existence of a "fast" longitudinal instability, is proposed. The equilibrium length is obtained by requiring that the growth rate be sufficiently larger than the rate of synchrotron oscillations. The theory is used to describe the bunch length data for SPEAR at $1.5 \mathrm{GeV}$. The low voltage and/or high current regime is dominated by a set of "low" frequency, low Q resonators [e.g., f $=320 \mathrm{MHz}$, $\Delta f(f w h m)=130 \mathrm{MHz}]$. To fit the observations in the high voltage and/or low current regime, a high frequency, low $Q$ impedance is required (e.g., $f=3.8 \mathrm{GHz}, \Delta f=1.0 \mathrm{GHz}$ ). The mechanism is mediated by the resistive component of the impedance. Thus, there is qualitative agreement with the observed distortion of the bunch tail. This is in contrast to the predictiuns of the potential well modela based on a reactive impedance source. These latter theories yield large distortions of the head of the bunch. The calculated power dissipated in the assumed sources by the given electron bunch is not inconsistent with estimates made for SPEAR. 


\section{Introduction}

We propose a mechanism for the anomalous electron bunch length based on the existence of a "fast" longitudinal instability. 1

We presume the presence of three time scales for electrons:

$$
\begin{aligned}
& \tau_{r}=\text { radiation damping time } \\
& \tau_{S}=\text { synchrotron oscillation period } \\
& \tau_{g}=\text { characteristic time for the "fast" longitudinal } \\
& \text { instability }\left(\alpha_{g}=1 / \tau_{g}\right. \text { is the instability growth } \\
& \text { rate). }
\end{aligned}
$$

The relationship between these times is

$$
\tau_{r} \gg \tau_{S} \gg \tau_{g}
$$

Let $\sigma_{l}$ be the rms bunch length as determined from the instability threshold and let $\sigma_{0}$ denote the rms equilibrium bunch length resulting from the balance of radiation damping and quantum excitations. ${ }^{2}$ Then, it follows that the actual steady state bunch length $\sigma_{A}$ will be given by

$$
\sigma_{\mathrm{A}}=\operatorname{Larger}\left\{\sigma_{\ell}, \sigma_{0}\right\}
$$

The fast instability characteristically develops as a bunch widening with unstable particles quickly losing energy. The role of the synchrotron motion is to mediate the instability overshoot and convert bunch widening into bunch lengthening.

If the initial bunch length is below threshold, instability causes growth and overshoot. Eventually the slower radiation damping will re-. duce the bunch area close to the threshold. At this point, further reduction is inhibited by Inclpient fast ingtability

Since $\sigma_{\ell}$ depends on the bunch current, this theory predicts a

1. E. Messerschmid and M. Month, "Theory for the Fast Blowup of Particle Bunches in Accelerators," submitted to Nucl. Instrum. Methods (1976).

2. M. Sands, SIAC Report No. 121, SLAC (1970). 
current threshold. For sufficiently small but nonzero current, we must have $\sigma_{\ell}<\sigma_{0}$, leaving the natural bunch length $\sigma_{0}$ as the observed one. If the current is increased sufficiently, then at some value $I_{t h}$, we have $\sigma_{l}=\sigma_{0}$. Above this current, $I>I_{t h}$, then $\sigma_{\ell}>\sigma_{0}$ and then the observed bunch length will be that corresponding to the instability threshold.

II. The "Fast" Longitudinal Instability ${ }^{3}$

Fium ubservations of proton bunches and computer simulations, single charged particle bunches have been shown to develop fast instabilities in the presence of sufficiently large longitudinal electric fields. Since growth rates are proportional to the frequency of the beam induced fields, these instabilities tend to be characterized by high frequencies, that is, high azimuthal mode numbers.

\section{Fast Instability Threshold for a Single Bunch}

It can be shown that for these fast instabilities, where $T_{g} \ll \bar{\tau}_{s} \ll \tau_{r}$, the threshold criterion for an unstable mode, $n_{o}$ ' can be expressed in terms of a threshold impedance, $Z_{t h}$, and a threshold rms energy spread, $\sigma_{E}$, by

$$
\left|\frac{z_{\text {th }}}{n_{0}}\right|=\frac{2 \pi \eta E}{e I} x_{E}^{2} \text {, }
$$

where $E$ is the particle energy, $I$ is the $d c$ current

$$
I=\operatorname{Nef}_{0},
$$

with $\mathrm{N}$ the number of particles in the bunch, $f_{0}$ the revolution

3. Much of this section follows ref. 1. Further references to the fast instability for protons can also be found in ref. 1 
frequency, $x_{E}$ is the relative rms energy spread,

$$
x_{E}=\sigma_{E} / E,
$$

and $\eta$ is the frequency slip factor,

$$
\eta=\frac{\mathrm{pdf}}{\mathrm{fdp}}=\left|\frac{1}{\gamma^{2}}-\frac{1}{\gamma_{\mathrm{tr}}^{2}}\right|,
$$

with $\gamma$ and $\gamma_{t r}$ the energy and transition energy in electron mass units. Since $Y \gg \gamma_{t r}, \eta$ can be expressed as

$$
\eta=\frac{1}{\gamma_{t r}^{2}} .
$$

In solving the dispersion relation, we have assumed Gaussian distributions in both energy and phase of the bunch. If $f_{0}$ is interpreted as corresponding to a full particle revolution, this implies we are considering the case of a single bunch.

The Effective Impedance Determining the Threshold for a Bunched Beam

The basic result for the fast instability of a single bunch is that a single azimuthal beam mode, say, $\mathrm{n}_{0}$, can excite fields at all modes, no The limit of this coupling is determined by the "bunch spectrum," i.e., the bunch lingth. The factor coupling a field of mode $n$ to a beam mode $n_{0}$ is found to be the usual coupling impedance of mode $n$. Writing

$$
\mathrm{m}=\mathrm{n}-\mathrm{n}_{\mathrm{o}},
$$

and denoting the coupling impedance of mode $n$ by $z_{m}$, it can be shown that the effective coupling impedance for Gaussian bunches is given by

$$
z_{\text {eff }}=\sum_{m=-\infty}^{\infty} z_{m} e^{-m^{2} \theta^{2}} r m s,
$$

where the exponential term represents the "bunch spectrum" limitation 
for a Gaussian bunch. The bunch length is represented in units of azimuthal angle (radians) by $\theta_{\text {rms }}$ :

$$
\theta_{\text {rms }}=2 \pi B_{r m s}=\frac{2 \pi \sigma_{l}}{c} \text {, }
$$

where $\sigma_{l}$ is the rms bunch length, $\mathrm{C}$ is the machine circumference, and $B_{\text {rms }}$ is the "rms bunching factor."

\section{The Threshold Bunch Length}

The threshold bunch length is determined by replacing $Z_{t h}$ in Eq. (2.1) with $Z_{\text {eff }}$ from Eq. (2.5):

$$
\left|\frac{z_{e}[f}{n_{0}}\right|=\left|\frac{z_{t h}}{n_{0}}\right|=\frac{2 \pi+\mid E}{e I} \cdot x_{E}^{2},
$$

where $Z_{\text {eff }}$ and $x_{E}$ are both functions of the bunch length, which, at threshold, we denote $\sigma_{\ell}$, or in terms of its angular length, $\theta_{\text {rms }}$.

The bunch length is related to the relative energy spread, $x_{E}$ through the synchrotron wave number, $\nu_{s}, b^{2}$

$$
\theta_{\text {rms }}=\frac{\eta}{\nu_{s}} x_{E}
$$

The current independent synchrotron wave number is given by

$$
\nu_{s}=\left[\frac{\eta h e V \cos \varphi_{s}}{2 \pi E}\right]^{\frac{1}{2}} \text {, }
$$

where $\mathrm{V}$ is the peak r.f voltage, $\mathrm{h}$ is the harmonic number, and $\varphi_{\varepsilon}$ is the stable phase angle, the latter determined by the rate at which energy must be provided to the beam by the rf system due to losses resulting from synchrotron radiation and the various dissipative forces in the ring. For large overvoltages, the effect on $\varphi_{S}$ due to synchrotron radiation energy loss can usually be neglected. 


\section{Case of a Purely Resistive Effective Impedance}

If we conjecture that the coupling impedance is dominated at high frequencies by resonators, it is not improbable that the sum of many such "low" Q elements can be simulated by a single strong "very 1ow" Q resonator. In this case, it can be easily seen that the effective impedance is purely resistive since the reactive component is an odd function of mode number. The resistive component, on the other hand, is even and, from Eq. (2.5) many modes can be seen to add up, limited only by the Gaussian cutoff factor.

Because the reactive component is negligible, we can use Eq. (2.8) relating bunch length and width, taking $\nu_{s}$ as the "natural" synchrotron wave number, from Eq. (2.9), determined only by the applied rf system.

\section{Effective Impedance for a Single Resonator}

Neglecting the reactive component, the impedance can be expressed by

where

$$
z_{m}=z_{R} \frac{a^{2}}{a^{2}+m^{2}}
$$

$$
a=\frac{n_{0}}{2 Q}
$$

$Q$ is resonator quality related to the resonance width, as in Eq. (2.10), $\mathrm{n}_{0}$ is the central resonator frequency, and

$$
\mathrm{Z}_{\mathrm{R}}=\mathrm{C}_{\mathrm{O}} \mathrm{Q},
$$

with $C_{0}$ an effective strength constant related to the strength of each individual "real" resonator and the manner in which they add up. 
For example, $M$ resonators in the $\mathrm{TM}_{01}$ mode, all at the same frequency and with unit transit time factor would yield a strength constant,

$$
\mathrm{C}_{\mathrm{O}}=111.4 \mathrm{M}
$$

The effective impedance in this case can therefore be written,

$$
\frac{z_{\text {eff }}}{n_{0}}=\frac{3}{2} C_{0} a \sum_{m} \frac{1}{a^{2}+m^{2}} e^{-m^{2} \theta^{2}} \text { rms }
$$

Note that in the limit, $a>\left(1 / \theta_{\text {rms }}\right)$, or $Q<\frac{1}{2} n_{0} \theta_{r m s}$, we can put $m=0$ in the Lurentz factor. I'hen, replacing the sum by an integration, we arrive at the limiting case of "low" $Q$,

$$
z_{\text {eff }} \rightarrow \frac{\sqrt{2 \pi} z_{R}}{\theta_{\text {rms }}}
$$

Defining the function

$$
F\left(a, \theta_{r m s}\right)=\sum_{m} \frac{a}{a^{2}+m^{2}} e^{-m^{2} \theta_{r u ̈ s s}^{2},},
$$

we have

$$
\frac{Z_{e E F}}{n_{0}}=\frac{1}{2} C_{0} F\left(a, \theta_{\text {rms }}\right)
$$

Equation for Equilibrium Bunch Length in the Presence of a

\section{Resonator}

Using Eqs. (2.8) and (2.9), then Eq. (2.7) becomes

$$
\left|\frac{z_{\text {eff }}}{n_{0}}\right|=\left|\frac{z_{t h}}{n_{0}}\right|=\frac{h V\left|\cos \varphi_{s}\right|}{I} \theta_{r m s}^{2} .
$$

If we assume that energy loss due to dissipative ring elements does not have too significant an impact on $\cos \varphi_{S}$, we $\operatorname{can}$ take $\cos \varphi_{S}$ to correspond 
to that value needed to compensate the energy loss due to synchrotron radiation alone.

Therefore, substituting Eq. (2.17) into Eq. (2.18), we have for the equilibrium bunch length the equation

$$
\frac{1}{2} C_{O} F\left(a, \theta_{r m s}\right)=\frac{h V\left|\cos \varphi_{s}\right|_{\theta^{2 m s}}^{2}}{I}
$$

This means that except for normalization, the threshold bunch length depends only on the two variables, $a=n_{0} / 2 Q$, and

$$
b=h V\left|\cos \varphi_{s}\right| / I
$$

If $U_{0}$ is the energy loss per turn due to synchrotron radiation, then we have for $\cos \varphi_{s}$,

where

$$
\left|\cos \varphi_{s}\right|=\left[1-\left(U_{0} / V\right)^{2}\right]^{\frac{1}{2}}
$$

with

$$
U_{0}=C_{\gamma} E^{4} / \rho
$$

$$
C_{Y}=\frac{4 \pi}{3} \frac{I_{e}}{E_{0}^{3}}=8.85 \times 10^{-5} \mathrm{~m}-\mathrm{GeV}^{3} \text {. }
$$

$\rho$ is the magnetic bending radius, $r_{e}$ is the classical electron radius and $E_{0}$ is the electron rest energy.

\section{Growth Rate for "Fast" Longitudinal Instability}

For a resistive impedance, it can be shown that the growth rate, $\alpha_{g}=1 / \tau_{g}$, can be expressed as

where

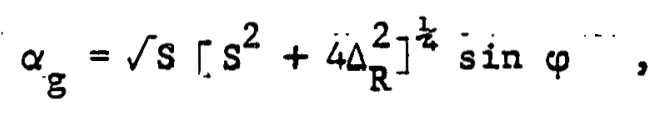

$$
\begin{aligned}
& \varphi=\frac{1}{2} \tan ^{-1} \frac{2 \Delta_{R}}{S}, \\
& S=\frac{x_{E} \eta n_{o} 2 \pi f_{o}}{\beta^{2}},
\end{aligned}
$$

and

$$
\Delta_{R}=\frac{\text { eIf }}{2 x_{E} E^{2}}\left(z_{\text {eff }}-z_{t h}\right)
$$


The quantity $\left(2 \Delta_{R} / S\right)$ can be put in the form

$$
\left(2 \Delta_{R} / S\right)=\frac{e I}{2 \pi \eta E x_{E}^{2}} \frac{\left(z_{\text {eff }}-z_{t h}\right)}{n_{0}} 。
$$

Using Eq. (2.1) this can be written in the simple form,

$$
\left(2 \Delta_{R} / S\right)=\frac{Z_{\text {eff }}}{Z_{\text {th }}}-1 \text {. }
$$

Thus, tho growth rate is given by

$$
\alpha_{g}=\frac{f_{0} e I}{x_{E}^{E}} z_{t h}\left[1+\left(\frac{z_{\text {eff }}}{z_{t h}}-1\right)^{2}\right]^{\frac{1}{4}} \sin \varphi \text {, }
$$

with

$$
\varphi=\frac{i}{2} \tan ^{-1}\left(\frac{z_{\text {eft }}}{z_{\text {th }}}-1\right)
$$

The rate of synchrotron oscillations is

$$
\alpha_{s}=\nu_{s} f_{0}
$$

Therefore, the growth rate in terms of the synchrotron oscillation rate is given by

$$
\frac{\alpha_{g}}{\alpha_{s}}=\frac{e I}{\nu_{s}^{x_{E}^{E}}} z_{t h}\left[1+(z-1)^{2}\right]^{\frac{1}{4}} \cdot \sin \varphi,
$$

or, using Eqs. (2.7), (2.8) and (2.9),

with

$$
\frac{\alpha_{\mathrm{g}}}{\alpha_{\varepsilon}}=2 \pi \mathrm{n}_{\mathrm{o}}{ }_{\mathrm{rms}}\left[1+(z-1)^{2}\right]^{\frac{3}{4}} \sin \varphi,
$$

$$
\varphi=\frac{1}{2} \tan ^{-1}(z-1) \text {. }
$$

For $z$ not too far from unity, we can write approximately,

$$
\frac{\alpha_{g}}{\alpha_{s}}=\pi n_{0} \theta_{r m s}(z-1)
$$


A consistency requirement for the "fast" longitudinal instability is that this ratio be sufficiently large, i.e.,

or roughly

$$
\alpha_{g} / \alpha_{s}>1
$$

$$
z-1>\frac{1}{\pi n_{0}^{\theta} \mathrm{rms}} \text {. }
$$

\section{Power Dissipation in Resistive Ring Elements}

The elements in the ring coupling to the beam through resistive impedances will cause the beam to lose energy. The power dissipation can be shown to be ${ }^{4}$

$$
P=\frac{1}{2} I^{2} \sum_{n} \operatorname{Re}\left(z_{n}\right) e^{-n^{2} \theta_{r m s}^{2}} .
$$

For the "single resonator," the resistive part is given in Eq. (2.10) and we have

$$
P=\frac{1}{2} I^{2} Z_{R} \sum_{n} \frac{a^{2}}{a^{2}+\left(n-n_{0}\right)^{2}} e^{-n^{2} \theta_{r m s}^{2}}
$$

III. Application to SPEAR Results at $1.5 \mathrm{GeV}^{(5,6)}$

\section{Theory}

Consider Eq. (2.34). It can be viewed as a correction term to the threshold expression Eq. (2.18) in determining the "equilibrlum" bunch length. Let us suppose that the fast instability will be effective at some particular growth rate: for example, when the growth rate is 4 times the synchrotron oscillation rate. Then for a given effective impedance, $z_{\text {eff }}\left(n_{0}\right)$, centered at the frequency, $n_{0}$, the stability criterion, including the frequency dependent correction

4.. See, for example, E. Ke11, CERN Report, CERN/ISR-TH/74-15 (1.974). 
term, can be expressed as

$$
\frac{z_{\text {eff }}\left(n_{0}\right)}{n_{0}}=\frac{z_{t h}}{n_{0}}\left(1+\frac{4}{\pi n_{0}^{\theta} r m s}\right) .
$$

Actually, we should include the observation of a reactive impedance in the form of a factor which takes account of the fact that the synchrotron frequency is a function of current. From the general observation on the current dependence of the synchrotron frequency, we should include a gorroetion factur, say $k(i)$. The actual synchrotron frequency is then related to the unperturbed synchrotron frequency $\nu_{\text {so }}$ by

$$
v_{s}(I)=k(I) v_{\text {so }}
$$

The stability criterion can then be written

$$
\frac{z_{\text {eff }}\left(n_{0}\right)}{n_{0}}=\frac{h V\left|\cos \varphi_{s}\right|}{I} \theta_{r m s}^{2} k^{2}(I)\left(1+\frac{4}{\pi n_{0} \theta_{\text {rms }}}\right),
$$

where we have used Eq. (2.18) for $z_{t h} / n_{0} \cdot$

From the observations of the synchrotron frequency at SPEAR, ${ }^{5}$ we can choose $k$ of the form

$$
k(I)=e^{-45 I_{0}^{2}}
$$

with I taken in units of A.

Recal1 that,

$$
z_{\text {eff }}=z_{R} \sum_{m} \frac{a^{2}}{a^{2}+m^{2}} e^{-m^{2} \theta_{\text {Ims }}^{2}}
$$

Therefore, we interpret Eq. (3.2) as follows: given a set of experimental conditions, i.e., V, I and E; and further given a particular

5. M. A. Allen, G. E. Fischer, M. Matera, A. P. Sabersky, and P. B. Wilson, Proc. IXth Inter. Conf. on High Energy Acce l, Stanford University, May 1974, p. 352. 
set of resonant structures described by $z_{R}, Q$ and $n_{0}$, then an equilibrium $\theta_{\text {rms }}$ is predicted by the stability criterion Eq. (3.2). If there is more than a single set of resonant structures, for example, 2 resonances at widely different frequencies, say $n_{1}$ and $n_{2}$, then the equilibrium $\theta_{\text {rms }}$ is the larger of the two, each being determined separately from Eq. (3.2). In other words,

$$
\theta_{r m s}=\max \left\{\theta_{r m s}\left(n_{1}\right), \theta_{r m s}\left(n_{2}\right), \theta_{r m s}(\text { natural })\right\} .
$$

To compare the theory with the experimental observations at SPEAR, we write Eq. (3.2) as

$$
G\left(\theta_{\text {rms }}, a, n_{0}\right)=\dot{g},
$$

where

and

$$
G\left(\theta_{\text {rüs }}, a, n_{o}\right)=\left(\frac{z_{\text {eff }}}{n_{o}}\right) /\left[\theta_{\text {rms }}^{2}\left(1+\frac{4}{\pi n_{0} \theta_{\text {rms }}}\right)\right] \text {, }
$$

$$
g=\frac{h V\left|\cos \varphi_{S}\right| k^{2}(I)}{I}
$$

Equation (3.5) can be inverted giving $\theta_{\mathrm{rms}}$ as a function of $g$ for a particular resonant impedance function.

We plot in Fig. 1 the function $\theta_{\text {rms }}$ (g) for two resonances, one centered at $320 \mathrm{MHz}$, the other at $3.8 \mathrm{GHz}$. There are two significant qualitative features demonstrated in these curves. First, $\theta_{\text {rms }}$ shows an extremely strong dependence on $g$ for low $g$ and secondly, $\theta_{\text {rms }}$ becomes rather insensitive to $\mathrm{g}$, at larger values. If the two curves cross as shown, it can then be seen how a low frequency resonance could dominate in the low $V$, high current regime (that is, relatively large bunch lengths) while under conditions of high voltage and/or low current, the dominant perturbing field is at much higher frequencies. 


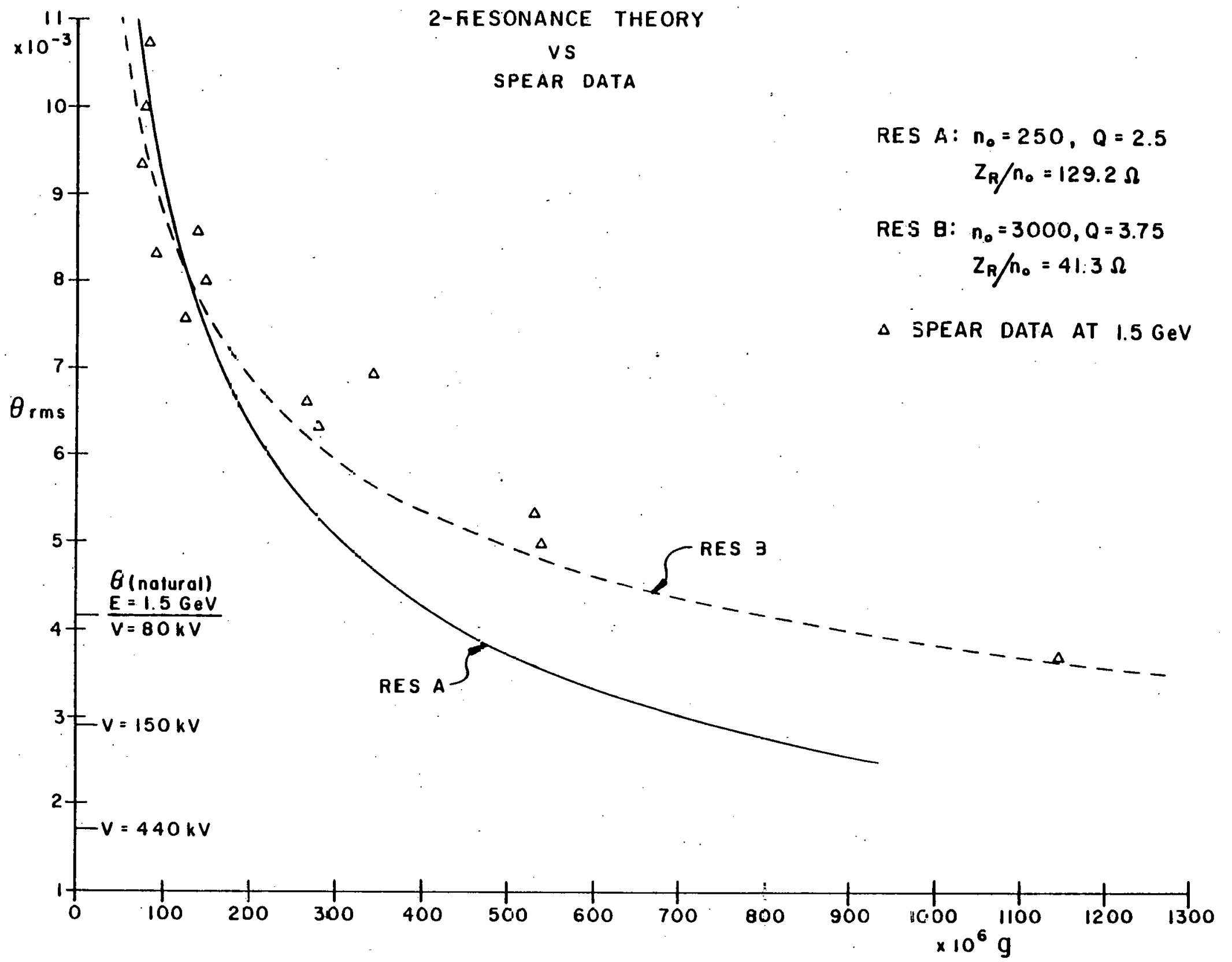

F1g. 1 Compartion of SPEAR data at $E=1.5 \mathrm{GeV}$ with 2-Resonance Theory. The rms bunch lengti, $\theta_{\text {rms }}$, versus the scaling parameter, $g$. 
The reason behind the choice of these frequencies is related to the implied power dissipation. In our theory, it is the resistive part of the impedance that is responsible for the bunch length. This means that the bunch length is directly related to the energy dissipated in the ring elements comprising the source of the perturbing impedance. We, therefore, have chosen parameters for the resonant impedances that give power dissipation in the range that is apparently observed at SPEAR。

\section{SPEAR Parameters, Observed Data and Comparison with 2-Resonance}

\section{Theory}

In Table I, we give the SPEAR parameters required for our analysis. The $1.5 \mathrm{GeV}$ data $^{5,6}$ are listed - in particular, the stability parameter $g$ and the corresponding bunch length observed.

To compute $g$, we use for the function $k(I)$, describing the current dependence of the synchrotron frequency, 5

$$
k^{2}(I)=e^{-90 I^{2}}
$$

with $I$ in amps. We also require the stable phase angle $\varphi_{s}$. Neglecting the energy loss due to resistive ring elements, $\cos \varphi_{S}$ can be obtained from Eq. (2.21).

The observed bunch lengths are plotted as a function of the $g$ parameter in Fig. 1. Keeping in mind that the equilibrium bunch length is determined from Eq. (3.4), the 2 -resonance model is in reasonably good agreement with the observed data. In fact, it might be argued that the single high frequency resonance is adequate.

6. A. M. Sessler, PEP Note 28, LBL (1973). 
TABLE I SPEAR I Data and Parameters

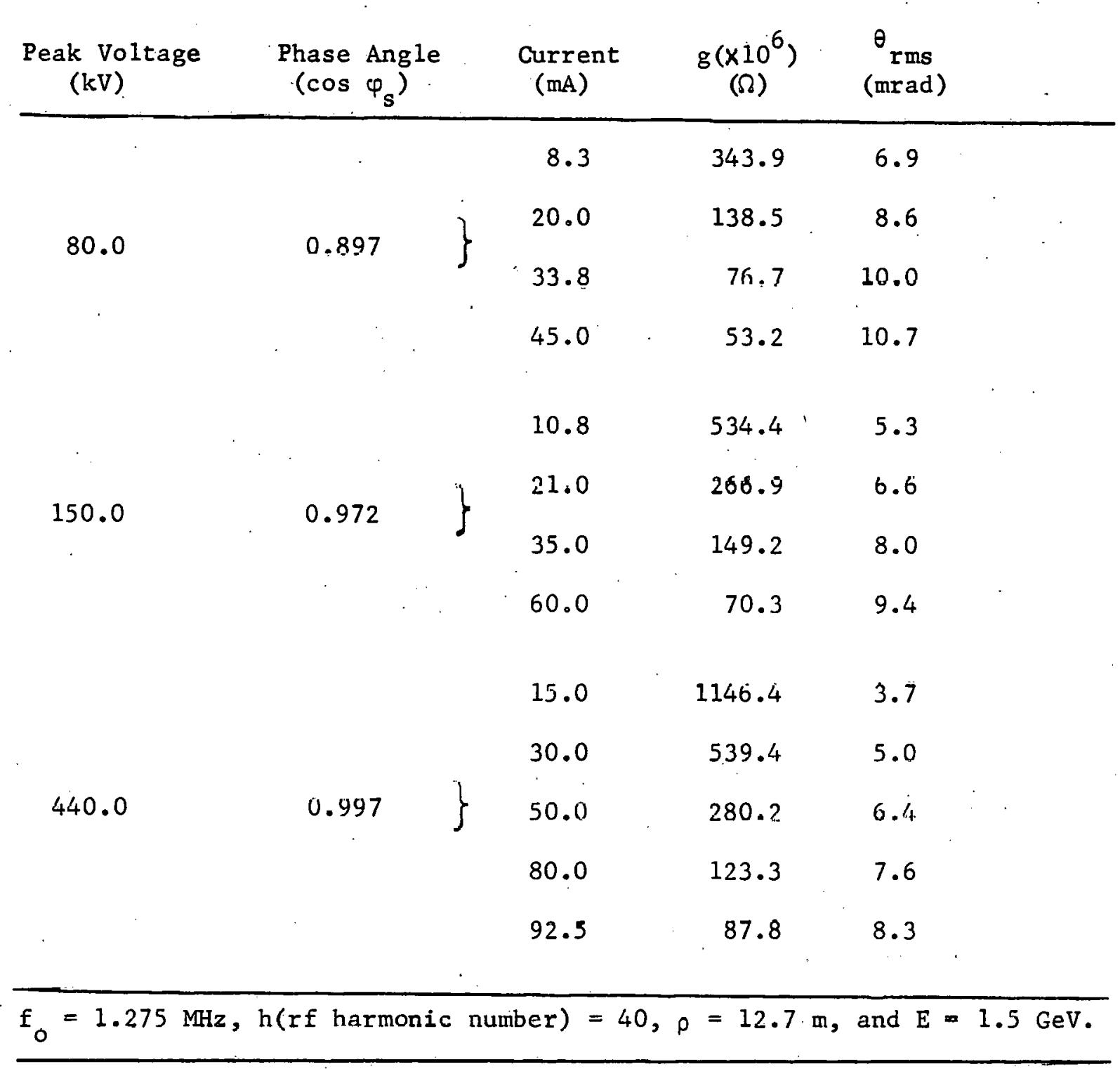


This is, of course, related to the scatter of the observed points in the $g-\theta_{\text {rms }}$ plane. This is presumably due to the uncertainty in the experimental parameters, although it is not completely inconceivable that the 2 variable picture is incomplete and more subtle effects underly this scatter. In any case, scaling with $g$ appears to be highly significant.

If we neglect the scatter, then the 2-resonance theory explains the general observation that low frequency resonators in SPEAR (the ferrite kickers) are indeed a source of bunch lengthening in the low voltage - high current (i.e., large $\theta_{\text {rms }}$ ) regime. The crossover reflects the fact that removal of these low frequency resonators will not affect the equilibrium bunch length for short bunches (i.e., in the high voltage and/or low current regime).

In Table II, we describe the parameters characterizing the two resonant impedance functions. The predicted power dissipation in the two resonant impedance sources, $P_{A}$ and $P_{B}$, are listed in Table III for the different experimental conditions.

\section{Conclusions}

We have described a mechanism for the anomalous electron bunch length in SPEAR based on the theory for a "fast" longitudinal insta: bility 1n Individual bunches. Attempting to fit the SPEAR data on bunch lengthening at $E=1.5 \mathrm{GeV}$, we require a high frequency, low $Q$ impedance. With an impedance peaking at $3.8 \mathrm{GHz}$ with a full width at half maximum of $1.0 \mathrm{GHz}$, we obtain agreement with the observations In the high voltage and/or low surrent regime. For larger bunch 
TABLE II Characteristics of Resonant Impedances

\begin{tabular}{|c|c|c|c|c|}
\hline & Symbol & Units & Res A & Res B \\
\hline Peak Impedance & $z_{R}$ & $\Omega$ & $3.23 \times 10^{4}$ & $1.24 \times 10^{5}$ \\
\hline Quality Fartor & $Q$ & - & 2.5 & 3.75 \\
\hline Mode Number & $\mathrm{n}_{0}$ & - & 250 & 3000 \\
\hline FWHM & $\Delta \mathrm{n}$ & - & 100 & 800 \\
\hline Central Frequency & $f$ & $\mathrm{GHz}$ & 0.32 & 3.8 \\
\hline Spread (FWHM) & $\Delta \mathbf{f}$ & $\mathrm{GHz}$ & 0.13 & 1.0 \\
\hline Peak Impedance & $\mathrm{z}_{\mathrm{R}} / \mathrm{n}_{\mathrm{O}}$ & $\Omega$ & 129.2 & 41.3 \\
\hline
\end{tabular}


TABLE III Power Dissipation into A and B Impedance Sources

\begin{tabular}{|c|c|c|c|}
\hline $\begin{array}{l}\text { Bunch Length } \\
\text { Orms (mrad) }\end{array}$ & $\begin{array}{c}\text { Current } \\
I(m A)\end{array}$ & $\begin{array}{l}\text { Power A } \\
\text { Watts }\end{array}$ & $\begin{array}{l}\text { Power B } \\
\text { Watts }\end{array}$ \\
\hline 6.9 & 8.3 & 22.2 & 19.1 \\
\hline 8.6 & 20.0 & 81.4 & 89.8 \\
\hline 10.0 & 33.8 & 169.4 & 219.6 \\
\hline 10.7 & 45.0 & 268.0 & 364.0 \\
\hline 5.3 & 10.8 & 66.3 & 42.3 \\
\hline 6.6 & 21.0 & 156.5 & 128.5 \\
\hline 8.0 & 35.0 & 282.8 & 294.7 \\
\hline 9.4 & 60.0 & 605.4 & 740.9 \\
\hline 3.7 & 15.0 & 239.6 & 119.0 \\
\hline 5.0 & 30.0 & 581.6 & 346.5 \\
\hline 6.4 & 50.0 & 972.4 & 758.5 \\
\hline 7.6 & 80.0 & 1676.7 & 1630.4 \\
\hline 8.3 & 92.5 & 1817.4 & 1977.8 \\
\hline
\end{tabular}

Res A: $Z_{R}=3.23 \times 10^{4} \Omega, n_{0}=250\left(f_{A}=0.32 \mathrm{GHz}, \Delta f_{A}=0.13 \mathrm{GHz}\right.$ ?

Res $B: Z_{R}=1.24 \times 10^{5} \mathrm{~s}, \mathrm{n}_{0}=3000\left(f_{B}=3.8 \mathrm{GHz}, \Delta f_{B}=1.0 \mathrm{GHz}\right)$ 
lengths (i.e., $\theta_{\text {rms }} \geqslant 8 \mathrm{mrad}$ ), almost any resonant impedance function gives a satisfactory fit. The presence of low frequency, low $Q$ resonators (e.g., with $f=320 \mathrm{MHz}, \Delta f=130 \mathrm{MHz}$ ) would be suitable. There are three qualitative features of our model which are consistent with the experimental facts. First, there is general agreement with the effect of the ferrite kickers. ${ }^{5,7}$ If we reduce the low frequency imperance, the theurry does predict a reduction in the bunch length in the low voltage - high current regime. For high voltages and/or low currents, the impact of the ferrite kicker removal actually diminishes. This is also predirter in our model. secondly, the mechanism presented here is predicated on the resistive component of the impedance. ${ }^{8}$ The small but noticeable elongation of the tail would tend to suggest just such a resistive impedance ${ }^{1,9}$ mechanism rather than one mediated by a reactive impedance, 7,10 in which case we would expect a large distortion of the heal of the bunch, 5,11 this latter affoct not belng observed. Finally, the power dissipated in the resistive impedances required in the theory is not inconsistent with observation. 5

7. F. J. Sacherer, PEP Note 45 and PEP Summer Study (1973).

8. A different approach, but also conjecturing that the source of bunch lengthening arises from a resistive impedance source is due to A. N. Lebodev, Prne. uf the inter. School of Physics, Varenna, Italy, Academic Press, 1971, p. 184. A resistive impedance has also recently been suggested by A. G. Ruggiero (DESY notes, private communication, 1976). See also, the "Strong Turbulence" approach, P.: J. Channel and A. M. Sesslex, LBL 4613 (197.5) submitted to Nuc $L$, Instrum. Methods.

9. H. G. Hereward, PEP Note 56 and PEP Summer Study (1973).

10. C. Pellegrini and A. M. Sessler, Nuovo Cimento 3A, 116 (1971).

11. A. Papiernik, M. Chatard-Moulin and B. Jecko, Proc. IX.th Inter. Conf. on High Energy Acce1., Stanford, Calif., 1974, p. 375; and E. Keil, SLAC Report, PEP 126 (1975). 